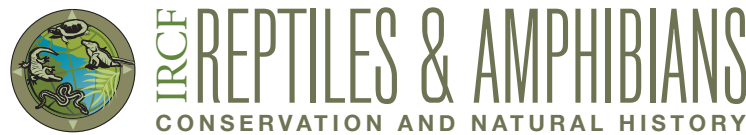

\section{Unexpected Iguanas: Emerging Iguanas and Conservation at the U.S. Consulate General Guayaquil, Ecuador ${ }^{1}$}

Julia C. Mellin

U.S. Department of State, Consulate General Guayaquil, Ecuador

$\mathrm{O}$ pening its eyes for the first time, the newborn Green Iguana (Iguana iguana) took in its surroundings: more than seven acres of rolling hills, a small forest of Ecuador's native trees-and dozens of other baby iguanas emerging from the sand nearby. After nearly 100 hours of digging, sifting, and sorting, a local biologist and his university students had transplanted more than 1,500 Green Iguana eggs from a single volleyball court at Consulate General Guayaquil to their new home at the University of Guayaquil.

${ }^{1}$ The original version of this article appeared in State Magazine's February 2020 issue (https://statemag.state.gov/2020/02/0220feat03/). Reprinted and adapted with permission. Changes involved adaptation to R\&A format and the insertion of scientific names.

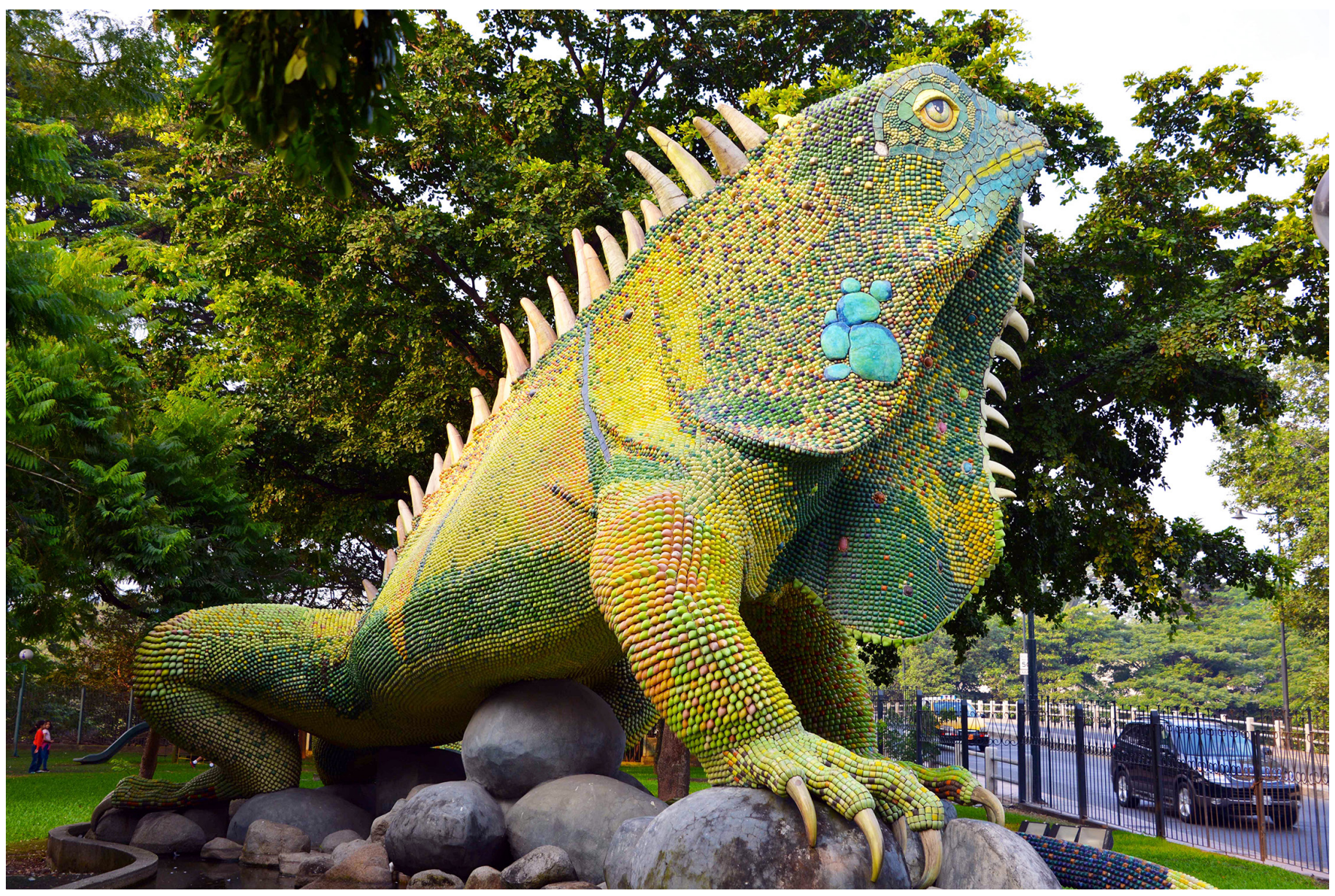

Fig. 1. This larger-than-life iguana statue is a famous Guayaquil city landmark, commissioned by the municipality to commemorate the animal and erected out of mosaic tile by artist Juan Marcelo Sánchez. Photograph courtesy of Flickr user talalbakr25. 


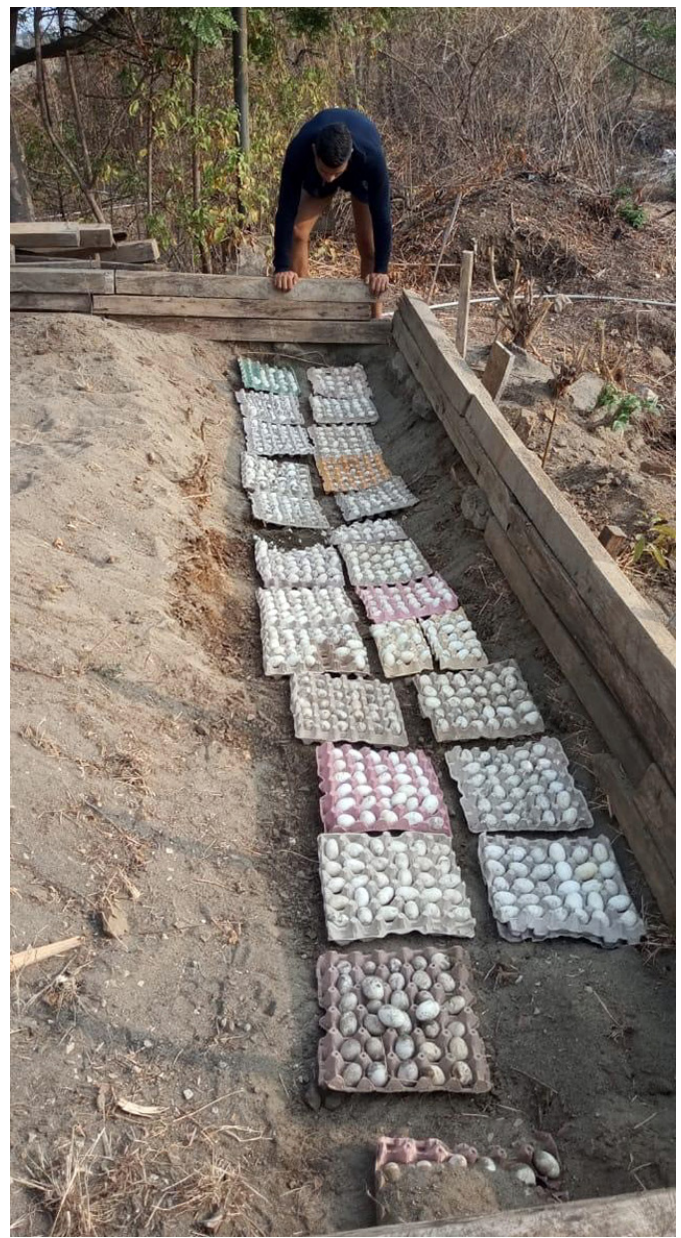

Fig. 2. A University of Guayaquil student prepares transplanted Green Iguana eggs for reburial at the University of Guayaquil in December 2019. Photograph by Antonio Torres Noboa.

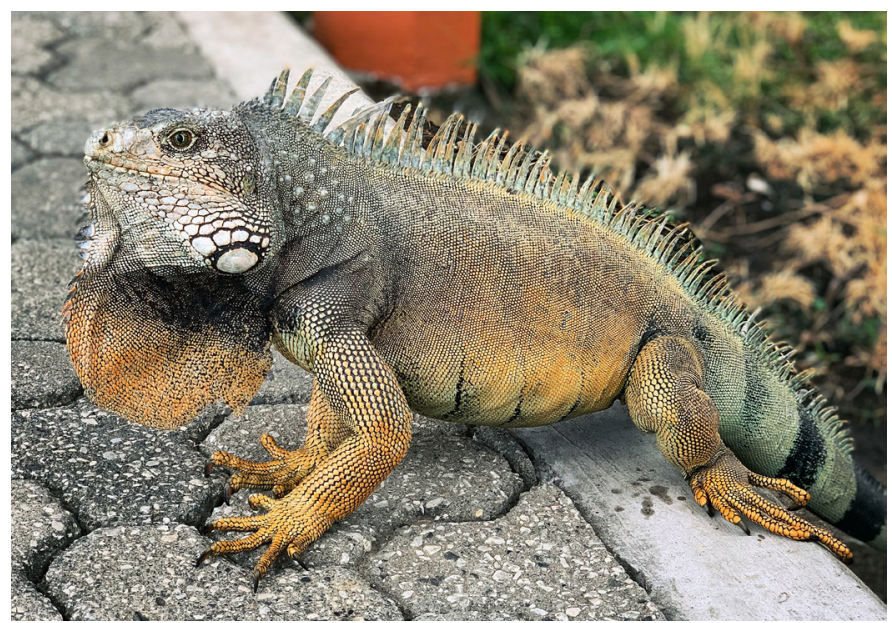

Fig. 3. A Green Iguana rests on an outdoor walkway at Consulate General Guayaquil in October 2019. Photograph by Julia Mellin.

Employees of Consulate General Guayaquil are accustomed to sharing their space with the local populace of Green Iguanas, many of whom have lived on consulate grounds since its groundbreaking in 2014. Not only is the consulate, dating to 1825 , one of the United States' oldest diplomatic posts in Latin America, but its campus has become an unexpected habitat for the Green Iguana in Ecuador. In a bustling industrial city of more than two million people, the sprawling green space of the consulate serves as a welcome respite for the lizard. Also known as the American Iguana, these reptiles spend their days napping by the koi pond, sunning themselves on the basketball court, or hoping for handouts next to the picnic tables.

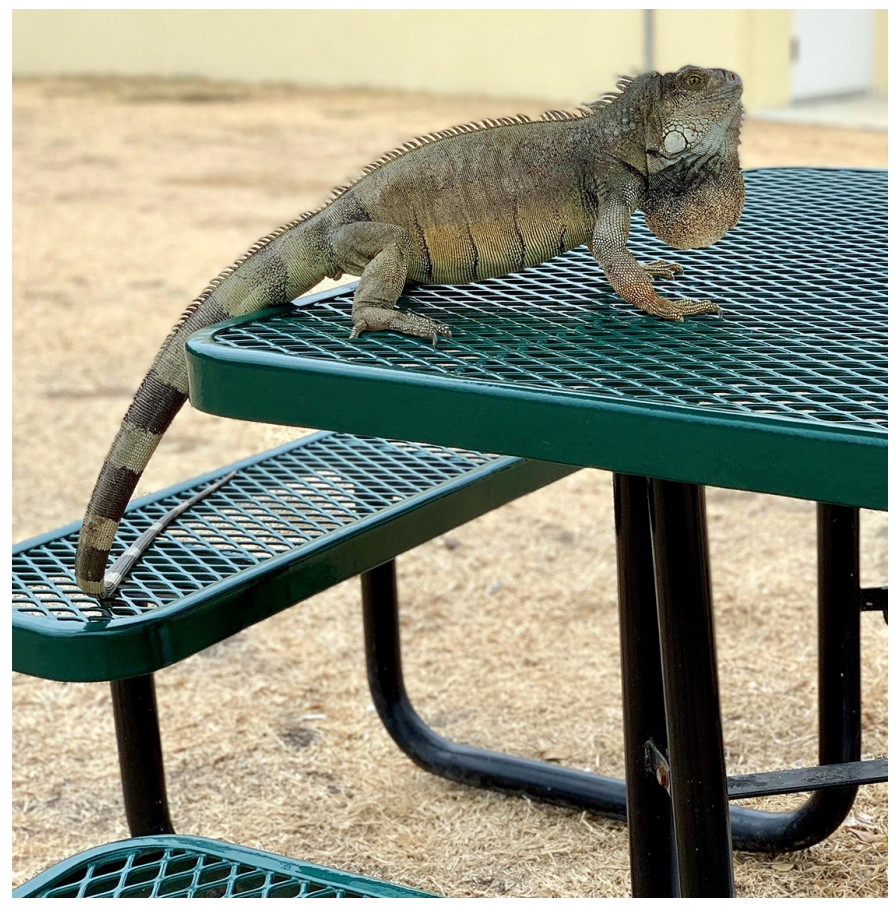

Fig. 4. A Green Iguana checks for scraps on top of an outdoor picnic bench at Consulate General Guayaquil in November 2019. Photograph by Julia Mellin.

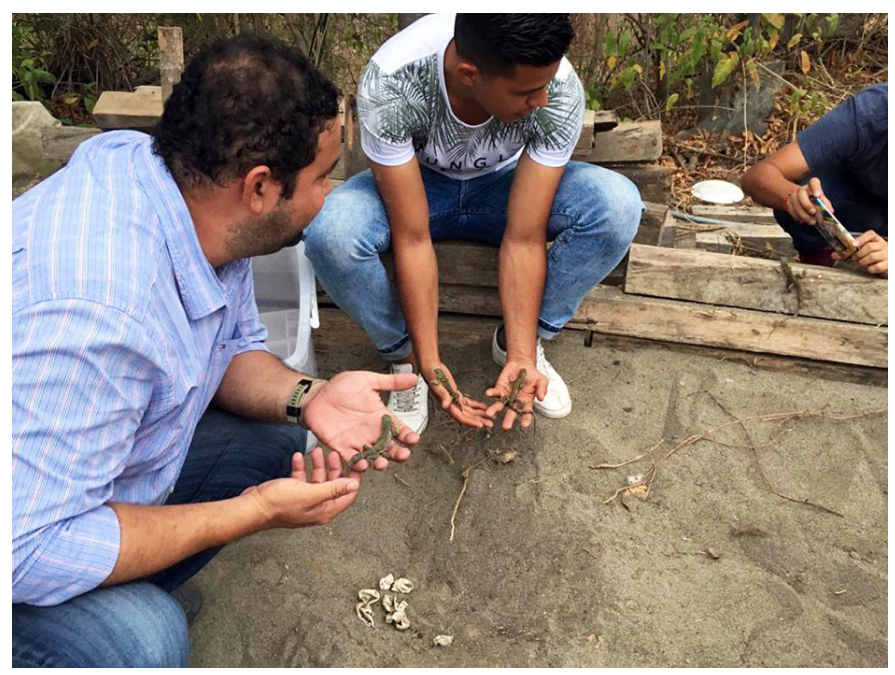

Fig. 5. Biologist Antonio Torres Noboa and his students examine newly hatched Green Iguanas at the University of Guayaquil in December 2019. Photograph by Pedro Ramos Montoya. 


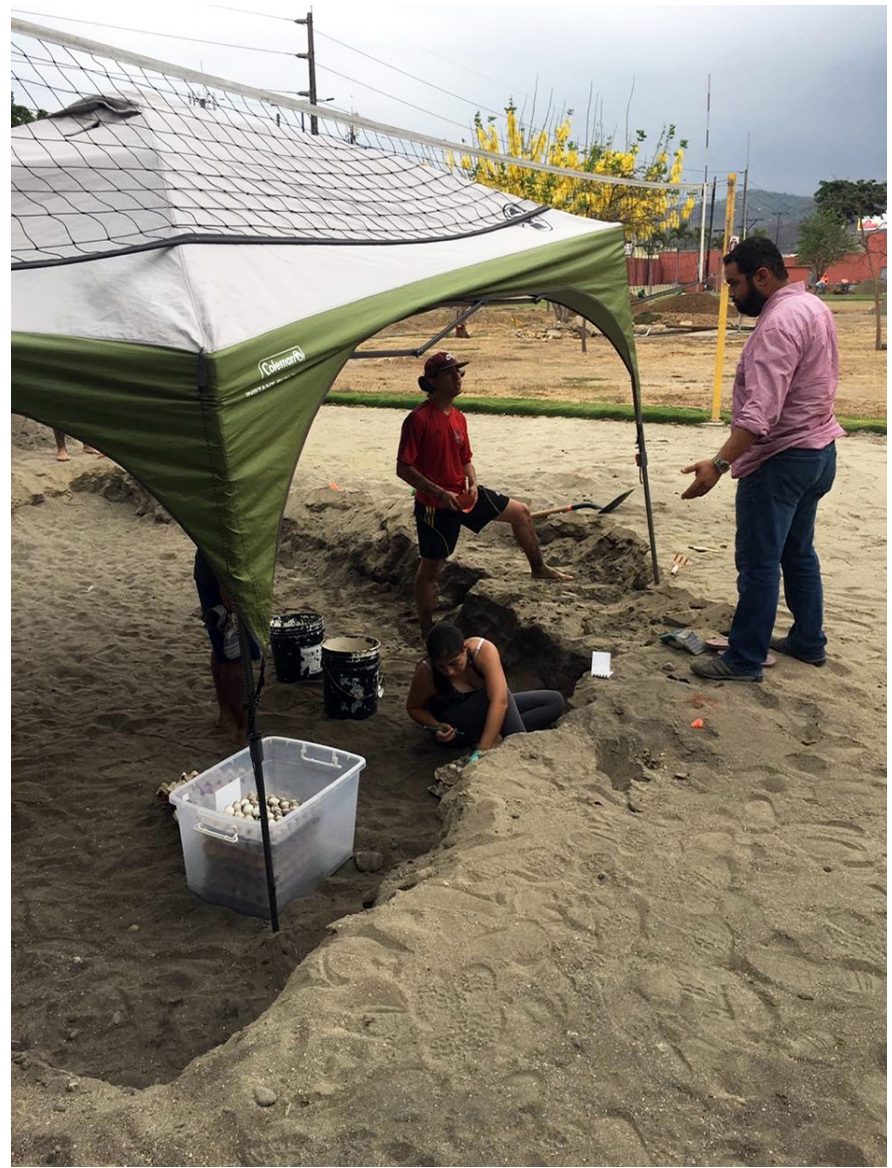

Fig. 6. Biologist Antonio Torres Noboa and his students digging for iguana eggs in the Consulate General Guayaquil volleyball court in December 2019. Photograph by Paolo R. Cassano.

In fall 2019, consulate staff realized just how appealing the environment was when iguanas began laying eggs in the consulate beach volleyball court. Seemingly emerging from nowhere, dozens of female iguanas dug deep tunnels into the sand and laid eggs in at least 65 buried nests. Every inch of the court was soon covered with drag marks left by iguana tails and countless interconnected tunnels hidden below the surface. The volleyball court, built to provide recreational space for consulate staff, turned out to provide a perfect setting for incubation. Green Iguana eggs need to incubate at a temperature between 85 and $91^{\circ} \mathrm{F}$ (approximately 29-33 ${ }^{\circ} \mathrm{C}$ ), and sand absorbs heat at a much higher rate than other types of soil-and is significantly easier to dig into.

Home to at least six species of iguanas, Ecuador is known for its progressive environmental policies. In the Galapagos Archipelago, several species of iguanas are considered threatened and are protected by law. While the Green Iguana is considered an invasive species in some parts of the world, in Guayaquil it is respected, and even revered. The city is covered in prominent statues and other public art commemorating the reptile, and even has a city park lovingly nicknamed "Iguana Park" that houses a large population. Guayaquileños grow up with Green Iguanas, which are as common in the

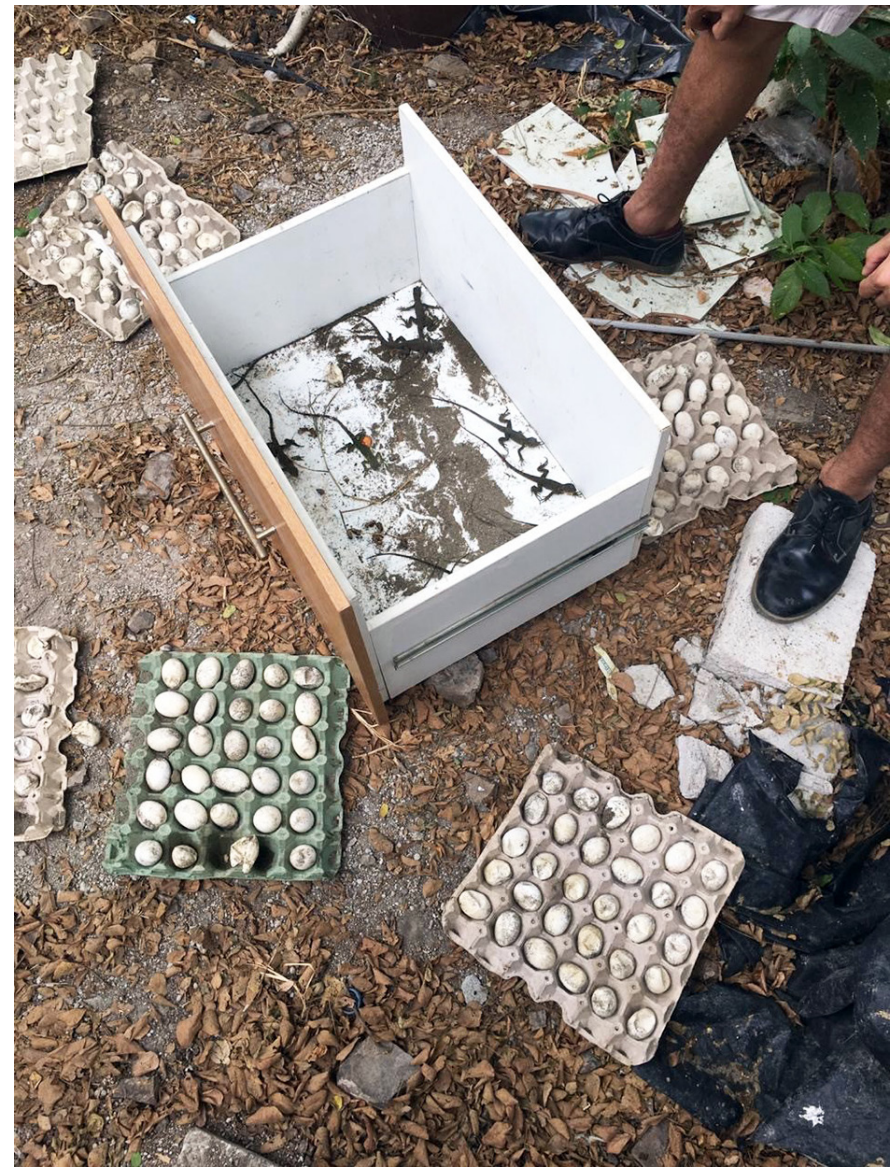

Fig. 7. Newly hatched Green Iguana babies and eggs at the University of Guayaquil in December 2019. Photograph by Antonio Torres Noboa.

city as squirrels or pigeons might be in certain parts of the United States.

While the consulate's iguana population has always thrived with ample tree shelter and natural food sources, the sudden influx of eggs raised new questions about supporting

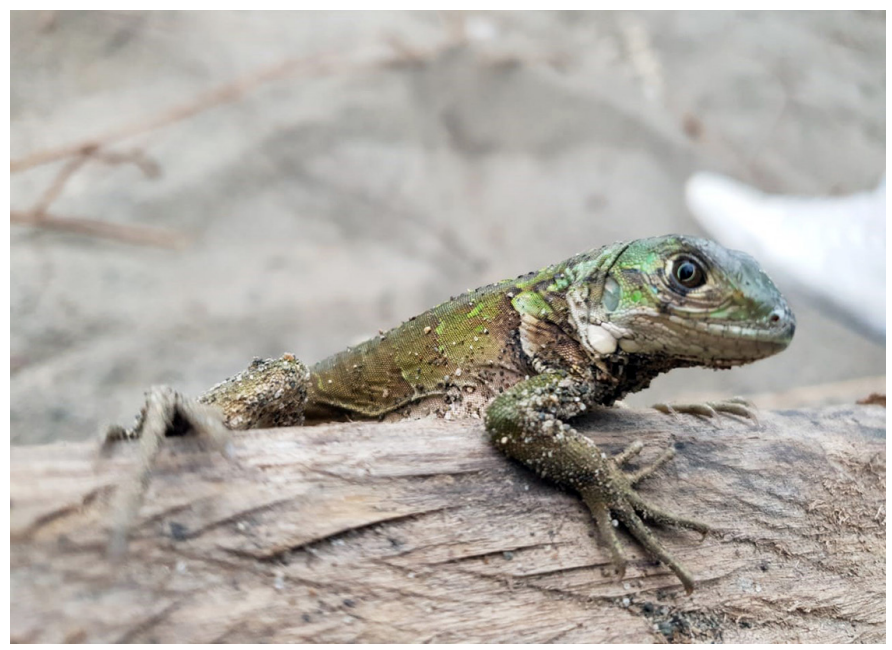

Fig. 8. A recently hatched Green Iguana leaves its sandy incubation site for the first time at the University of Guayaquil in December 2019. Photograph by Antonio Torres Noboa. 


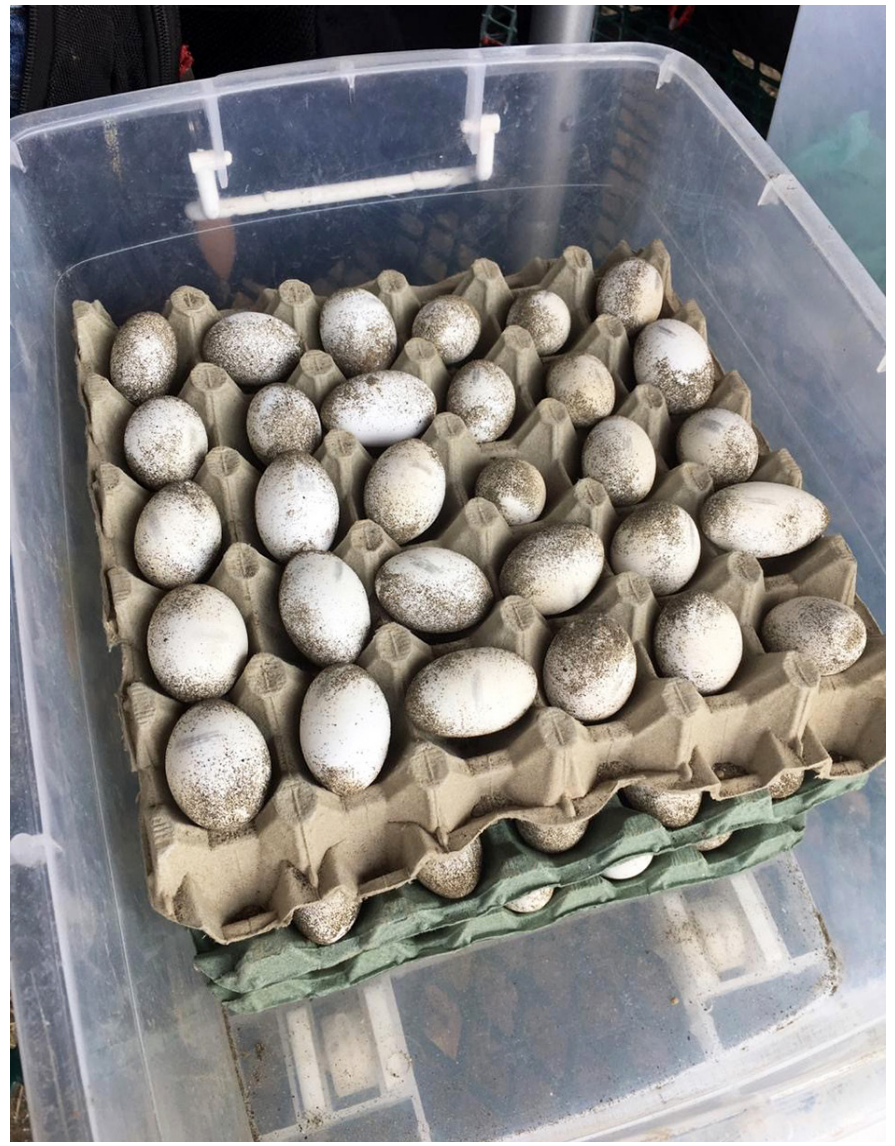

Fig. 9. Green Iguana eggs removed from Consulate General Guayaquil await transport and reburial at the University of Guayaquil in December 2019. Photograph by Antonio Torres Noboa. so many newborns. With the assistance of the consulate's environmental conservation and awareness group, management and facilities staff reached out to biologist Antonio Torres Noboa for help in safely relocating the eggs. Torres Noboa's team discovered numerous sites around the consulate where iguanas had attempted to dig below the surface to lay eggs but had been thwarted by rocky soil. However, the beach volleyball court's soft soil allowed the iguanas to burrow with ease. Consulate Facilities Manager Kevin Gately explained, "When we created the volleyball court, we created life!"

In order to determine the consulate's capacity to house thousands of new baby iguanas, Torres Noboa calculated the quantity of vegetation cover provided by the consulate's trees. Iguanas depend on edible plants and trees to provide sustenance and shelter. Essentially, every 50 square meters of vegetation cover can support one iguana. Based on Torres Noboa's estimates, the consulate would have been overrun if the eggs laid in the volleyball court had been allowed to hatch there. The consulate currently houses approximately 200 Green Iguanas, and like most reptiles, iguanas do not raise their young, but lay eggs and then leave them to fend for themselves when born. As adults, they come back to the same location where they were born to lay their own eggs. For this reason, it was vital that the iguanas be born somewhere they could live out their lives and reproduce without fear of overpopulation.

Torres Noboa worked closely with the consulate's facilities management team to excavate the eggs and rebury them

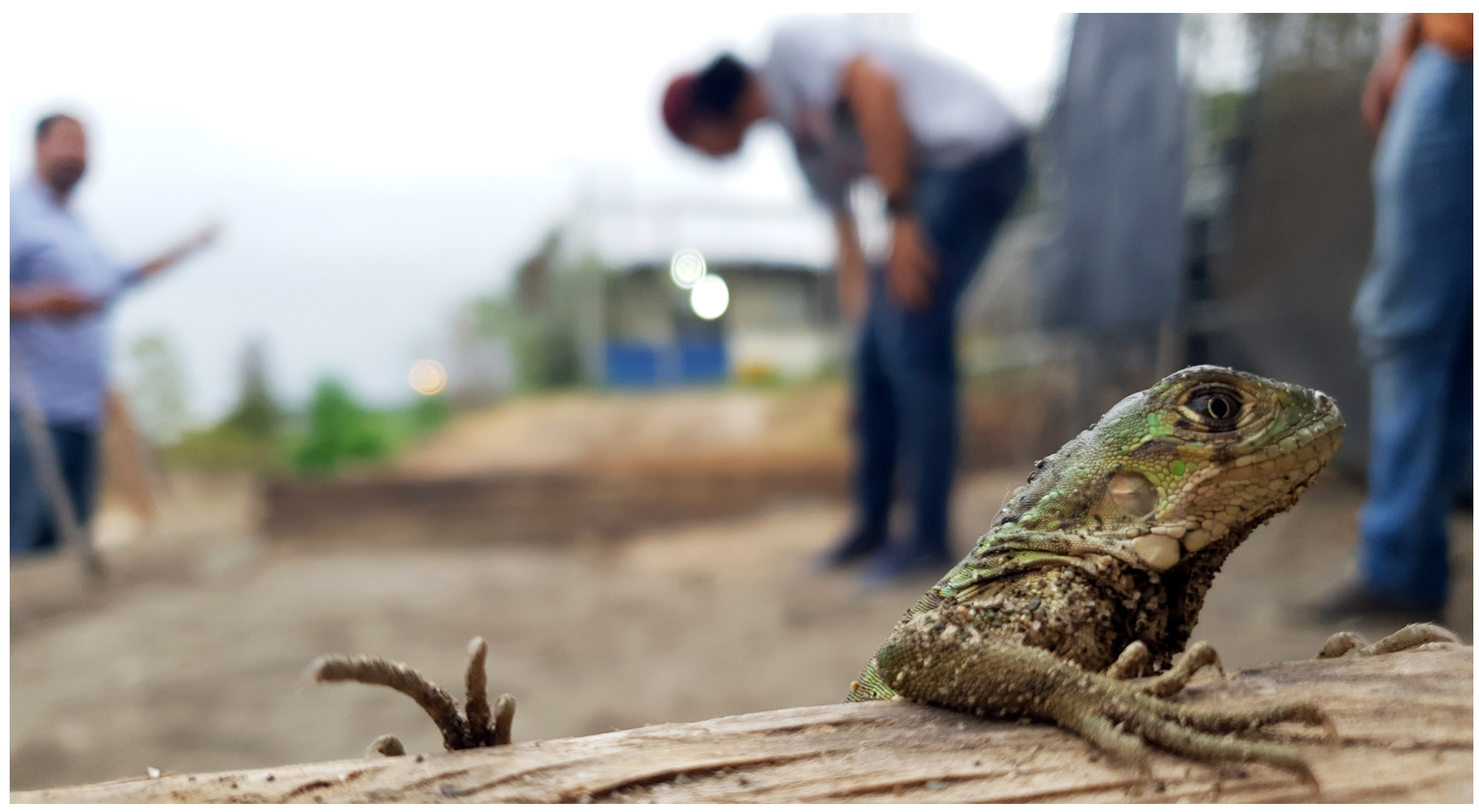

Fig. 10. A recently hatched Green Iguana leaves its sandy incubation site for the first time at the University of Guayaquil as biologist Antonio Torres Noboa and his students look on in December 2019. Photograph by Antonio Torres Noboa. 


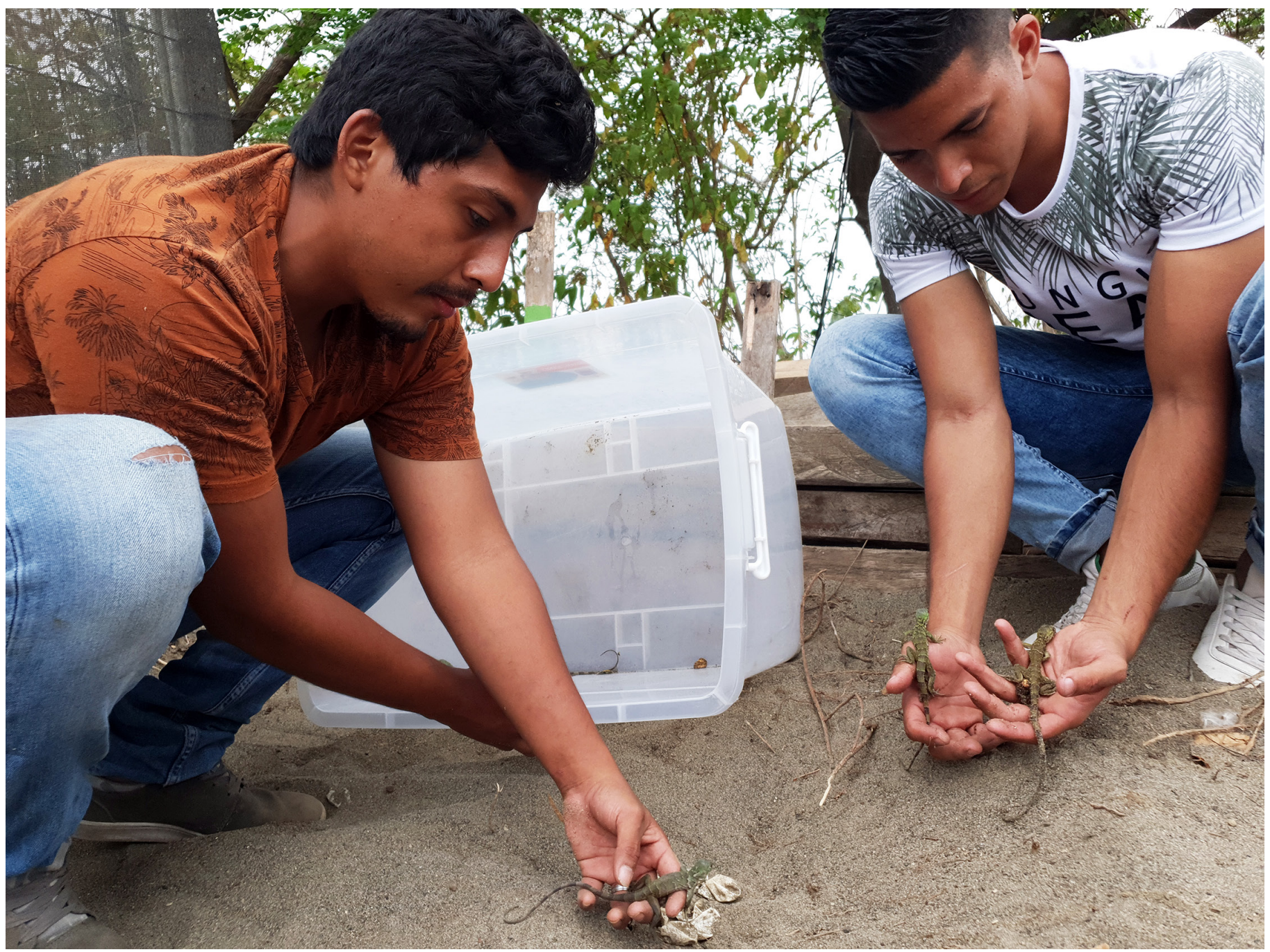

Fig. 11. University of Guayaquil students examine newly hatched Green Iguanas at the University of Guayaquil in December 2019. Photograph by Antonio Torres Noboa.

in a larger and more suitable environment on the University of Guayaquil's natural sciences campus. In addition to a large bed of sand-constructed to mimic the volleyball court's ideal conditions - the campus erected several incubation chambers for any eggs that appeared underdeveloped. Already home to other iguanas, chipmunks, and native Ecuadorian trees, the campus is an ideal environment for the newly hatched babies. Within days of being rehomed, more than 250 of the eggs had hatched. After several more weeks, over 400 more would eventually hatch.

Over the span of barely six weeks, Consulate General Guayaquil staff learned more about the reproductive practices, behavior, and habitat of Green Iguanas than they ever could have imagined. For its part, the volleyball court will now be treated annually with vinegar, a natural iguana repellent that will biodegrade, doing no harm to the environment while ensuring that eggs are not laid there again in the future. 